\title{
Atherosclerotic plaque regression and HMG-CoA reductase inhibition potential of curcumin: An integrative omics and in-vivo study
}

\author{
Priyanka Riyad, Ashok Purohit, Sen Karishma, Heera Ram* iD \\ Department of Zoology, Jai Narain Vyas University, Jodhpur, India.
}

\begin{tabular}{l}
\hline ARTICLE INFO \\
\hline Article history: \\
Received on: April 06, 2021 \\
Accepted on: May 20, 2021 \\
Available Online: January 07, 2022
\end{tabular}

\section{Key words:}

Curcumin, HMG-CoA

reductase, molecular docking, ADMET, atherosclerotic plaque, Castelli indexes

\begin{abstract}
The current study was carried out to evaluate the potential of curcumin against the progression of atherosclerosis and cholesterol biosynthesis by incorporating the combined data of in-vivo assessments and integrative omics examinations. The high fat diet and supplementation of cholesterol powder caused significant alterations in the lipid profile as well as hypercholesterolemia indices. The induced hypercholesterolemia promoted progression of atherosclerotic plaque with the occurrence of foam cells in a bulged structure. Simultaneously, the treatments of curcumin and atorvastatin caused significant reductions in total cholesterol, low density lipoprotein cholesterol, and very low-density lipoprotein cholesterol, as well as hypercholesterolemia indices of Castelli Risk Index-I and II and atherogenic indices. Accordingly, the treatments of curcumin and atorvastatin caused significant regressions in atherogenic plaque area, total wall area, and increased lumen volume. Subsequently, molecular docking showed significant interactions of curcumin and atorvastatin with $\beta$-Hydroxy $\beta$-methylglutaryl-CoA reductase, which were depicted by bonding energy, number of $\mathrm{H}$-bonds, and bond length. Accordingly, the absorption, distribution, metabolism, excretion, and toxicity and toxicity data revealed significant druggability of curcumin along with supportive analysis of Brain Or IntestinaL EstimateD-Egg prediction of gastrointestinal absorption. Thus, it can be illustrated that curcumin has significant potential to promote regression in atherosclerotic plaque and subside the cholesterol biosynthesis by inhibition of HMG-CoA reductase, as indicated by the outcomes.
\end{abstract}

\section{INTRODUCTION}

Curcumin is one of the most explored bioactive ingredients of turmeric from the early Vedic civilization till today for different aspects of therapeutics for several ailments [1,2]. Even the healing therapeutic properties have been reported in the ancient civilization and are picturized in several epics of Ramayana and Mahabharata. Accordingly, modern science has explored numerous medicinal properties of curcumin such as anti-inflammatory, anti-lipid peroxidation, antidiabetic, anticancer, antioxidant, hepatoprotection, and cardioprotective efficacies, as well as hypocholesterolemic properties, through different parameters by the existing facilities $[3,4]$. Curcumin, a polyphenol, has been shown to target various signaling molecules, although indicating activity at the cellular level, which has assisted in the establishment of several health benefits [5].

*Corresponding Author

Heera Ram, Department of Zoology, Jai Narain Vyas University, Jodhpur, India.E-mail:hr.zo@jnvu.edu.in
Accordingly, a search carried out on PubMed (National Library of Medicine and National Center for Biotechnology Information) was filtered using the keywords abstract, free full text, full text, associated data, books and documents, clinical trial, meta-analysis, randomized controlled trial, review, and systematic review from the period of $2000 / 1 / 1$ to $2021 / 1 / 1$, which showed 339 results causally related to the health benefits of curcumin. Beside this, the consumed curcumin by itself does not lead to the accompanying health assistances due to its deprived bioavailability, which is principally owing to poor absorption, rapid metabolism, and rapid elimination [6a plantderived polyphenolic compound, naturally present in turmeric (Curcuma longa]. Therefore, the current study planned to evaluate the potential of atherosclerotic plaque regression and inhibition of $\beta$-Hydroxy $\beta$-methylglutaryl-CoA (HMG-CoA) reductase of curcumin by using integrative omics and in-vivo approaches because cardiovascular disease is governed by atherosclerosis leading to internal cholesterol biosynthesis and progression of atherosclerotic plaque. This attempt was carried out by taking input from previous studies and incorporating it in the recent view. The study incorporated integrative omics parameters along with in-vivo results and concluded 
that curcumin has the capability of regressing atherosclerotic plaque and subsiding the cholesterol biosynthesis through the inhibition of HMG-CoA reductase.

\section{MATERIALS AND METHODS}

\subsection{Procurement of Curcumin and Chemicals}

Curcumin (C1386) was procured from Sigma-Aldrich, Mumbai, India, by local chemical suppliers. Accordingly, the cholesterol powder, stains, reagents, and other necessary chemicals were obtained from Loba Chemie, which were of chemical grade.

\subsection{Data Retrieval and Grid Preparation of Ligand and Protein}

The extremely determined crystallographic structure of HMGCoA reductase (PDB ID 1DQA) was obtained from a Protein Data Bank in the PDB format. Subsequently, the three-dimensional structure of curcumin was downloaded from the PubChem database (PubChem CID 689043). The protein was further processed for the removal of water molecules and correcting the chain integration using AutoDock tools to extract the cocrystallized ligand inhibitor. A three-dimensional structure of the curcumin compound was downloaded from the PubChem database (PubChem CID 689043) [7].

\subsubsection{Molecular docking}

Curcumin was exposed to molecular docking analysis by AutoDock Vina using HMG-CoA reductase as the receptor protein $[8,9]$. The molecular interactions of HMG-CoA reductase were analyzed by using PyMol and LIGPLOT. The retrieved protein of HMG-CoA reductase was further processed for the removal of water molecules and correcting the chain integration using AutoDock tools to extract the co-crystallized ligand inhibitor. Subsequently, curcumin (ligand) was processed using PyMol and hydrogen atoms were added to the structure using the AutoDock tools. Molecular interactions, conformations of the ligand, and their binding energies were acquired in the analysis.

\subsubsection{ADMET assessment}

absorption, distribution, metabolism, excretion, and toxicity (ADMET) assessments were carried out by using SwissADME to evaluate the absorption, distribution, metabolism, and excretion (ADME), physiochemistry, drug-likeness, pharmacokinetic profile, and medicinal chemistry friendliness properties of the phytocompounds for drug development [10,11]. Curcumin was evaluated based on two filters: Lipinski's rule of five and the ability to pass the blood-brain barrier (BBB). Lipinski's rule of five helps to differentiate between drug and drug-like molecules. Lipinski's rule states that an ideal drug molecule should have a molecular mass below $500 \mathrm{Da}$, the number of hydrogen bond donors should be $\leq 5$, and number of hydrogen bond acceptors should be $\leq 10$, along with high lipophilicity or a partition coefficient of $\leq 5$. A compound with these traits would be able to pass the BBB if the number of hydrogen bonds present is in between 8 and 10, and no acidic groups are present in the molecule. The total polar surface area (TPSA) indicates the bioavailability of the drug molecule as per Weber's rule. A TPSA $\leq 140 \AA$ indicates good oral bioavailability.

\subsubsection{Gastrointestinal absorption-a Brain Or IntestinaL EstimateD (BOILED)-Egg model}

The BOILED-Egg permeation method is a predictive model that works with accuracy by computing the polarity and lipophilicity of small molecules and generates clear graphical outputs [12]. This tool is used to estimate various stages of the drug discovery process. Two pharmacokinetic characteristics play a crucial role, i.e., the prediction of gastrointestinal passive absorption and the permeability of the BBB.

\subsubsection{Toxicity prediction}

ProTox-II online tool was used for toxicity prediction to design the development of the drug. ProTox-II incorporates the prediction of the various toxicities of the chemical which can be measured qualitatively as binary (active or inactive) in terms of endpoints such as acute toxicity, hepatotoxicity, carcinogenicity, immunotoxicity, mutagenicity, cytotoxicity, adverse outcome (toxicology in the 21 st century) pathways, and toxicity targets [11]. It can be further measured quantitatively as $50 \%$ lethal dose $\left(\mathrm{LD}_{50}\right)$ values. Toxicity classes (I-VI) can also be predicted based on the toxic doses.

\subsection{Experimental Animals and Design}

Male albino rabbits (1.25-1.5 kg; aged 1 year and above) were obtained from the Lala LajpatRai University of Veterinary andAnimal Sciences, Hisar, Haryana, India. The animals were acclimatized in spacious cages under standard environmental conditions (12-hours light/dark cycle with maintained at $23^{\circ} \mathrm{C}$ temperature) for 10 days. The animal were fed a standard diet recommended the veterinarian and water ad libtum. The experimental protocols were approved by the departmental Institutional Animal Ethical Committee (IAEC NO. 1646/GO/ERe/S/19/CPCSEA). The experimental animals were categorized into four groups for the experimental duration of 2 months (15 days for induction of hypercholesterolemia and 45 days for treatments) as follows:

Group 1: Control; treated with only distilled water for 45 days;

Group 2: Hypercholesterolemic diet for 45 days;

Group 3: Treatment with curcumin ( $35 \mathrm{mg} / \mathrm{kg} /$ day) for 30 days after induction of hypercholesterolemia for 15 days;

Group 4: Treatment with atorvastatin $(0.25 \mathrm{mg} / \mathrm{kg})$ for 30 days after induction of hypercholesterolemia for 15 days.

\subsection{Induction of Hypercholesterolemia}

Healthy male rabbits were obtained for the development of animal models. Hypercholesterolemia was induced through the administration of a high fat diet and supplementation of cholesterol powder at the dose of $500 \mathrm{mg} / \mathrm{kg}$ body weight/day mixed with 5 $\mathrm{ml}$ of coconut oil for 15 days $[13,14]$.

\subsection{Dose and Duration}

Curcumin was administered by oral cannulation at $35 \mathrm{mg} / \mathrm{kg}$ body weight/day dissolved in olive oil for 45 days as per the literature [15]. Atorvastatin (dose $0.25 \mathrm{mg} / \mathrm{kg}$ body weight/day with distilled water) was used as the standard hypocholesterolemic drug [16]. 

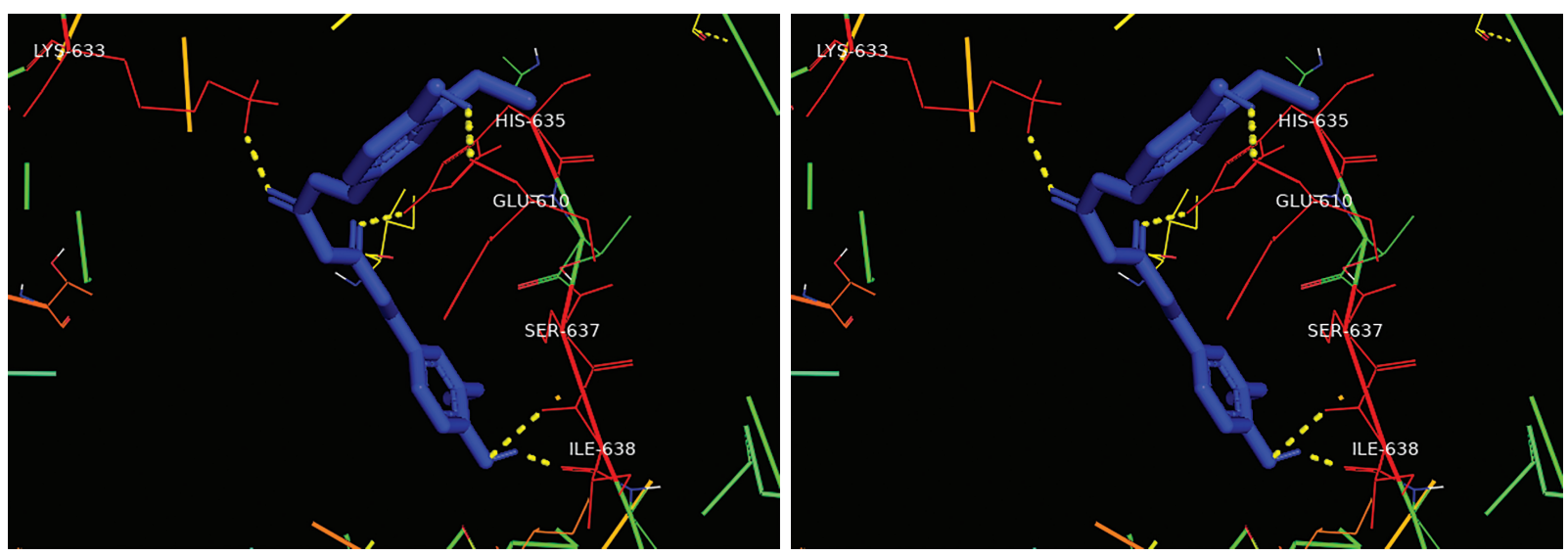

Figure 1: $(\mathrm{a}$ and $\mathrm{b})$ Molecular interaction between ligand curcumin and HMG-CoA reductase studied through docking analysis.

\subsection{Criteria of Observation}

Overnight-fasted animals were autopsied under mild anesthesia as per the recommendations given by the vegetarian by following the American Veterinary Medical Association guidelines after completion of the 45-day experiment period [17]. Blood was collected through direct cardiac puncture and separated serum stored at $-20^{\circ} \mathrm{C}$ for biochemical analysis. Accordingly, the vital organs (liver, kidney and heart) and aortas were removed, rinsed, weighed, and fixed in $10 \%$ formalin for histological preparations [18].

\subsection{Serum Biochemistry}

Parameters of the lipid profile and dyslipidemia indices [Castelli Index I and II and atherogenic index of plasma (AIP)], such as

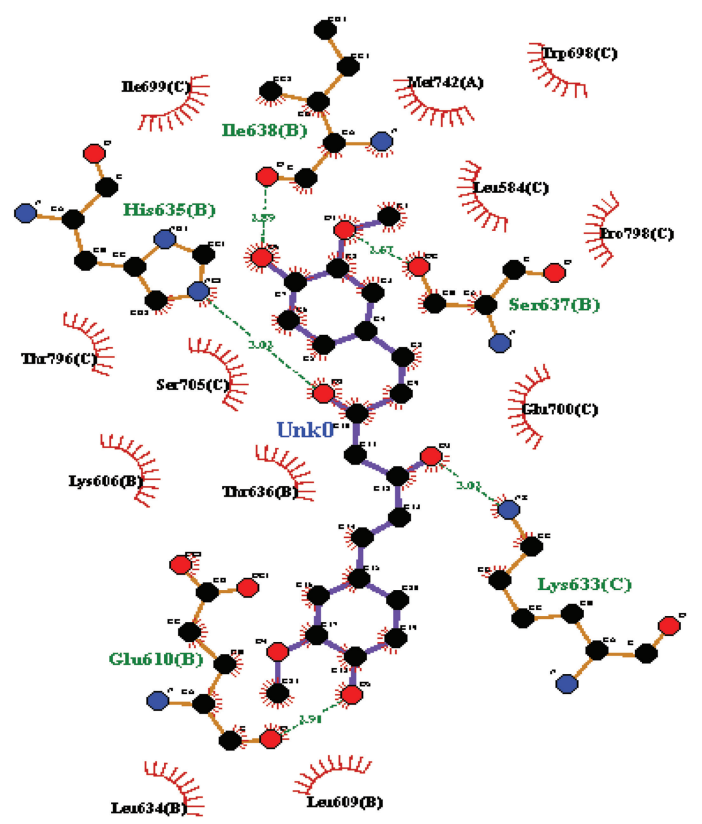

Figure 2: LIGPLOT diagram demonstrating the ligand-protein interactions. Residues involved in the ligand bonds are shown in thick lines (purple); residues involved in hydrogen binding with the ligand (black bonds) with thin orange bonds along with their hydrogen bonds with the length in middle (olive green); and residues involved in non-bonded interactions or hydrophobic contacts between protein and ligand are the red spikes. total cholesterol (TC) [19], triglyceride (TG) [20], low density lipoprotein (LDL)-cholesterol and HDL-cholesterol [21], and other biochemical parameters were estimated by using standard methods. The lipid profile [22] and dyslipidemia indices were calculated by using the routine formulas [23].

\subsection{Statistical Analysis}

Mean \pm standard error of the mean showed the results of biochemical analysis and body and organ weights; statistical variances were estimated by using analysis of variance and posthoc mean separation tests.

\section{RESULTS}

\subsection{Molecular Docking}

Significant interactions were made by HMG-CoA reductase through its catalytic grove which comprises residues from 426 to 888. Accordingly, curcumin presented as a potent ligand by providing the H-bonds and functional groups (Figs. 1A, 1B and 2). Curcumin was found to have strong binding energies with the main catalytic site residues indicating that they would inhibit the target protein in comparison to the standard drug (Table 1).

\subsection{ADMET Pharmacokinetics Predictions}

Pharmacokinetic (ADMET) analysis of curcumin indicated an ideal drug profile, further conforming the Lipinski rule of five as no

Table 1: Molecular docking of curcumin against the target enzyme of $\mathrm{HMG}-\mathrm{CoA}$ reductase.

\begin{tabular}{ccc} 
Ligand & Curcumin & Atorvastatin \\
$\begin{array}{c}\text { Binding energy } \\
(\mathrm{Kcal} / \mathrm{mol})\end{array}$ & -7.7 & -6.2 \\
No. of H-bonds & 5 & 2 \\
& & \\
Bond length $(\AA)$ & $\begin{array}{c}3.02,2.91,3.02,2.89, \\
2.67\end{array}$ & $3.97,3.33$ \\
& $\begin{array}{c}\text { Lys633, Glu610, } \\
\text { Interacting residues }\end{array}$ & His635, Ile638, Ser637 \\
\hline
\end{tabular}


Table 2: ADMET pharmacokinetics predictions of curcumin by SwissADME.

\begin{tabular}{ccc} 
Compound & Curcumin & Atorvastatin \\
MW & 368.38 & 558.64 \\
ilogP & 3.27 & 3.81 \\
ClogP & 3.03 & 4.99 \\
HBA & 6 & 6 \\
HBD & 2 & 4 \\
nHB & 8 & 10 \\
TPSA & 93.06 & 111.79 \\
N violations & 0 & 1 \\
Drug-likeness & Yes & Yes \\
Filter L/B & L & \\
\hline
\end{tabular}

$\mathrm{MW}=$ molecular weight; ilogP = partition coefficient; $\mathrm{Clog} \mathrm{P}=$ consensus $\log \mathrm{P}$; HBA $=$ hydrogen bond acceptor; $\mathrm{HBD}=$ hydrogen bond donor; $\mathrm{nHB}=$ number of hydrogen bonds; TPSA $=$ total polar surface area; violations $=$ number of violations; Filter $\mathrm{L}=$ Lipinski's rule of five and $\mathrm{B}=$ blood brain barrier

violations were shown by the compound. TPSA was found below $140 \AA$, indicating good cell membrane permeability (Table 2).

\subsection{Toxicity}

For toxicity studies, properties such as hepatotoxicity, carcinogenicity, immunotoxicity, mutagenicity, cytotoxicity, $\mathrm{LD}_{50}$, and toxicity class were predicated. It belonged to class 4 and was inactive as a toxicant but showed immunotoxicity (Table 3).

\subsection{BOILED-Egg Predictions}

Curcumin has good potential to cross HIA (the passive human gastrointestinal absorption) but does not have the ability to cross the $\mathrm{BBB}$ and predicted non-substrate of the P-glycoprotein (PGP) (Fig. 3).

\subsection{Body and Organ Weight}

Non-significant changes were observed in the body and organ weight of all treated groups except liver. A significant increase in the weight of liver was observed in the hypercholesterolemic group (Table 4).

\subsection{Lipid Profile and Dyslipidemia Indices}

TC, LDL-C, TGs, and very low-density lipoprotein (VLDL)-C were increased 16-fold $(p \leq 0.001)$ in cholesterol-fed

Table 3: In-silico toxicity study of curcumin by ProTox-II.

\begin{tabular}{ccc} 
Compound & Curcumin & Atorvastatin \\
Hepatotoxicity & Inactive & Active \\
Carcinogenicity & Inactive & Inactive \\
Immunotoxicity & Active & Inactive \\
Mutagenicity & Inactive & Inactive \\
Cytotoxicity & Inactive & Inactive \\
Predicted $\mathrm{LD}_{50}(\mathrm{mg} / \mathrm{kg})$ & 2,000 & 5,000 \\
Predicted toxicity class & 4 & 5 \\
\hline
\end{tabular}

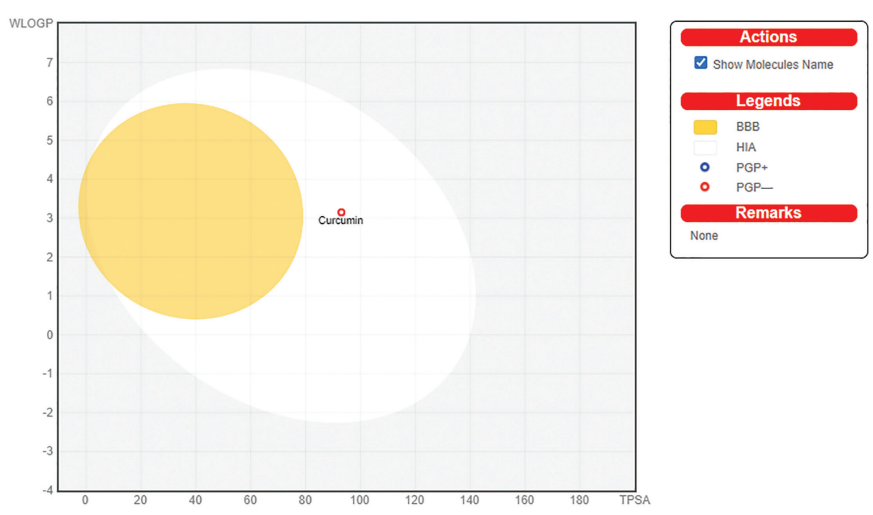

Figure 3: Predicted BOILED-Egg model diagram of the curcumin compound (SwissADME Web tool).

hypercholesterolemic rabbits, while administration of curcumin at the dose of $35 \mathrm{mg} / \mathrm{kg}$ body weight/day for 30 days showed significant reductions $(p \leq 0.001)$ in TC, LDL-C, VLDL-C, and TGs (Figs. 4 and 5).

\subsection{Histopathology of Aorta}

The aortal wall of hypercholesterolemic rabbits exhibited three layers of tunica intima, tunica media, and adventitia along with a bulged structure of atherosclerotic plaque. The plaque area employed with foam cell and degenerated elastin fibers (Fig. 6B). Atorvastatin- and curcumin-treated groups showed significant reductions in atherosclerotic plaque and increases in lumen of aorta (Fig. 6C and 6D).

\subsection{Planimetric Study}

The high fat diet and supplementation of cholesterol powder made alterations in the aortal wall area, lumen volume, and plaque area

1200

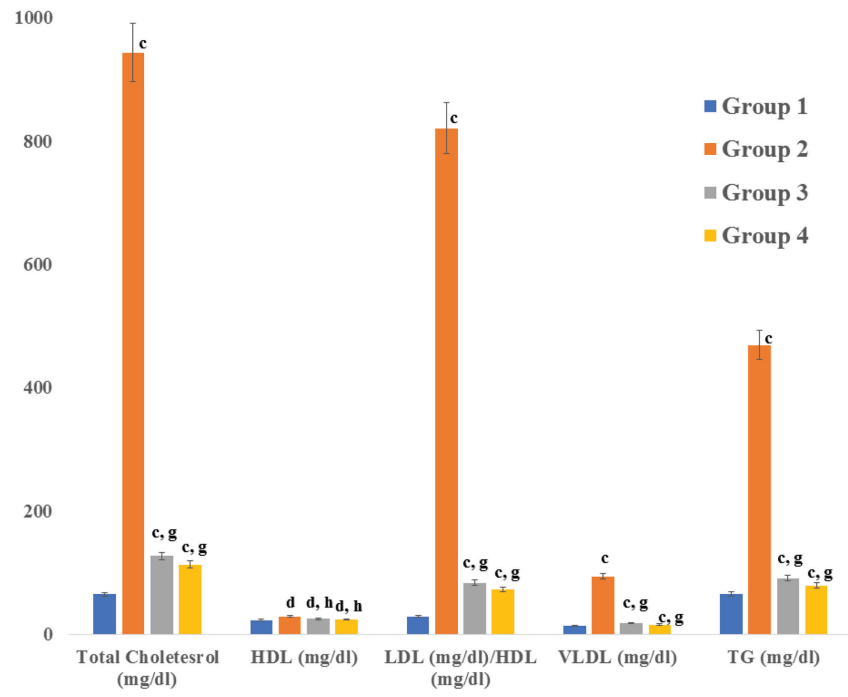

Figure 4: Lipid profile of control and treatment groups. 
Table 4: Effect of curcumin treatment on body and organs weight in high cholesterol-fed diet-induced hypercholesterolemic rabbits.

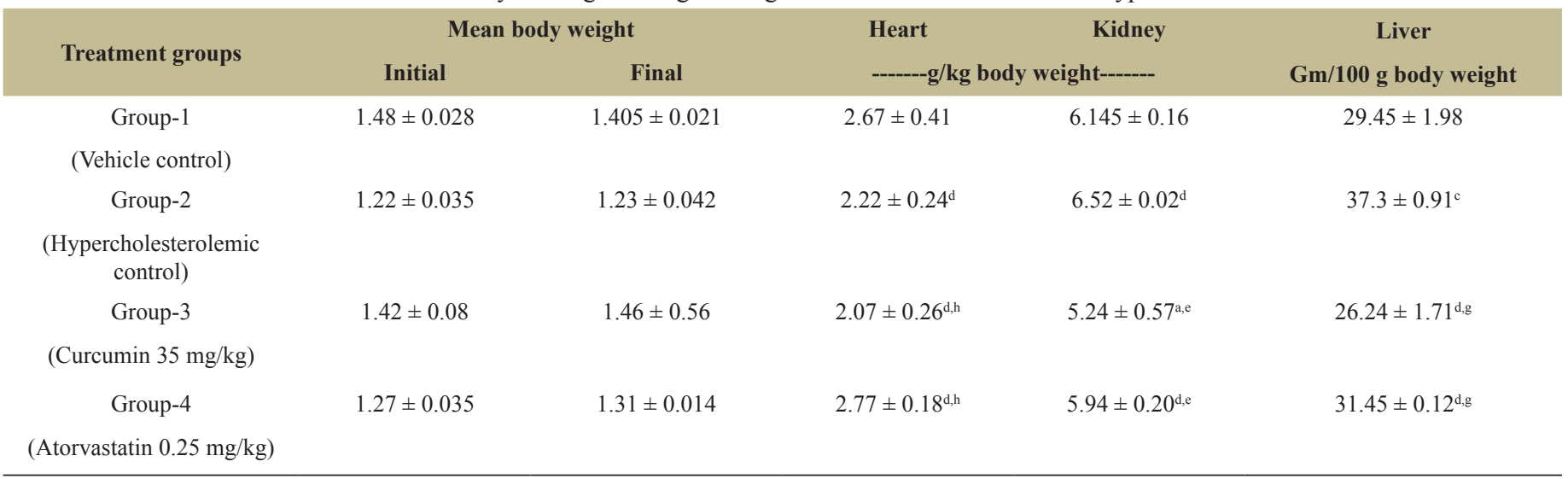

Gr. 2-4 compared with Gr. 1; Gr. 3-4 compared with Gr. $2 ; p \leq 0.05=\mathrm{a} ; p \leq 0.05=\mathrm{e} ; p \leq 0.01=\mathrm{b} ; p \leq 0.01=\mathrm{f} ; p \leq 0.001=\mathrm{c} ; p \leq 0.001=\mathrm{g}$; Non-significant $=\mathrm{d}$; Non-significant $=\mathrm{h}$.

(Fig. 7), whereas the treatments of curcumin and atorvastatin caused significant regressions in plaque area, total wall area, and lumen volume of aorta.

\section{DISCUSSION}

The small molecule phytocompounds have potential to interact with proteins and enzymes which make a lead for the development of therapeutics of several ailments [24]. These ideas are widely used in Ayurveda, traditional Chinese medicines, Yunani medicines, and other tribal centers oriented toward local medicines which are supported by long experiences of traditional wisdom and utilization of indigenous resources for health concerns. Consequently, curcumin is one of the compounds which is used in several medicines and serve as active pharmaceutical ingredient (API) based on its peculiarity of exhibiting the potent

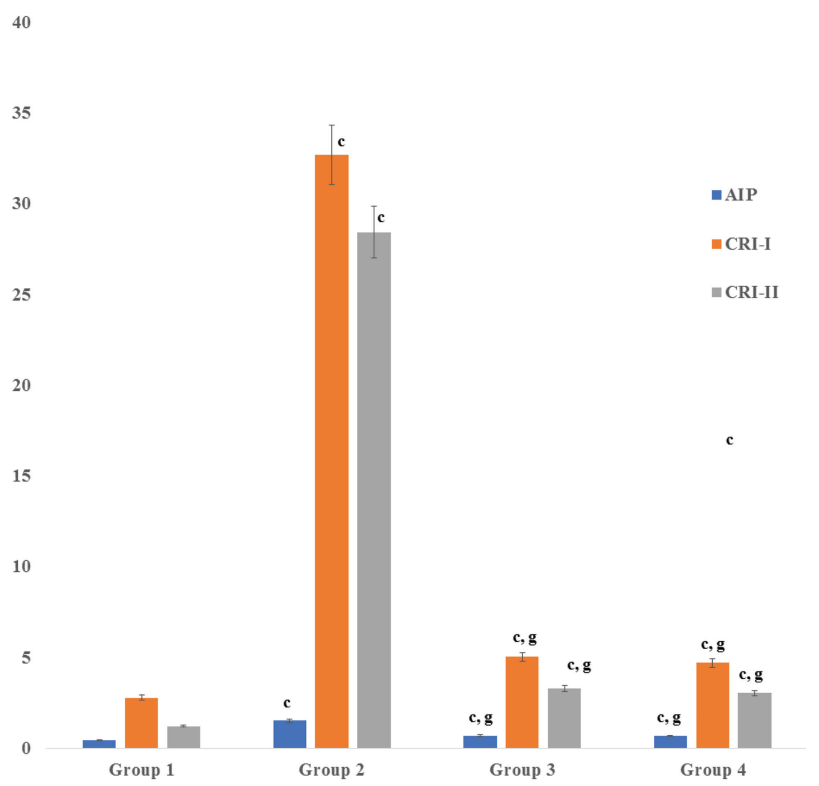

Figure 5: Dyslipidemia indices of AIP, Castelli Risk Index-I, and Castelli Risk Index-II of the control and treatment groups. functional groups $[2,25]$. The current study showed a similar kind of observation about the interaction with target protein of HMG-CoA reductase by revealing significant binding energy in comparison to the standard drug of atorvastatin. Accordingly, the total number of H-bonds and bind length was accomplished by using complementary, geometrically matched receptors and functional groups [26]. The additional interactive characteristics are enhancing and subsiding the bioavailability and bioactivity of test compounds. The bioavailability, bioactivity, and drug-likeness are represented by the absorption, distribution, metabolism, excretion, and toxicity data which can be predicted by the ADMET examination [27]. The current study shows the suitable prediction

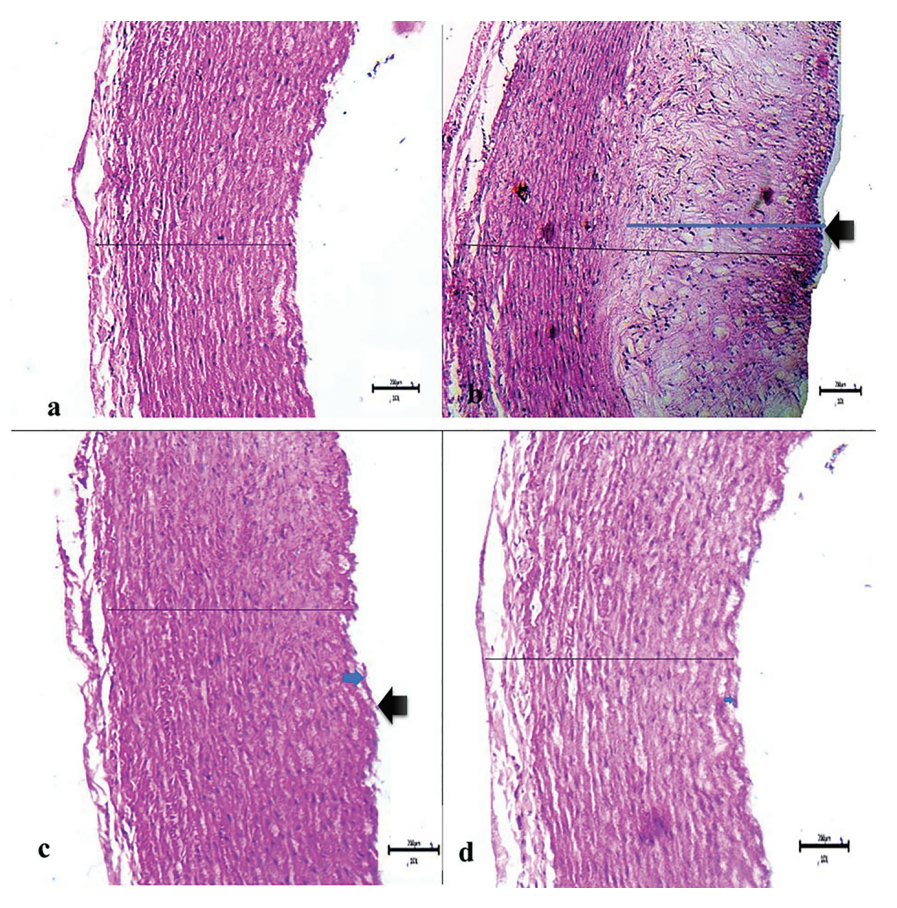

Figure 6: Histology of aorta of various treatment groups $(200 \times$ hematoxylin and eosin). (a) Histoarchitecture of intact aorta with a compact wall.

(b) Hypocholesterolemic aorta exhibiting the bulging structure of plaque (thick arrow). (c) Aorta of curcumin treatment with restorated histoarchitecture. (d) Aorta of atorvastatin treatment with restorated histoarchitecture. 


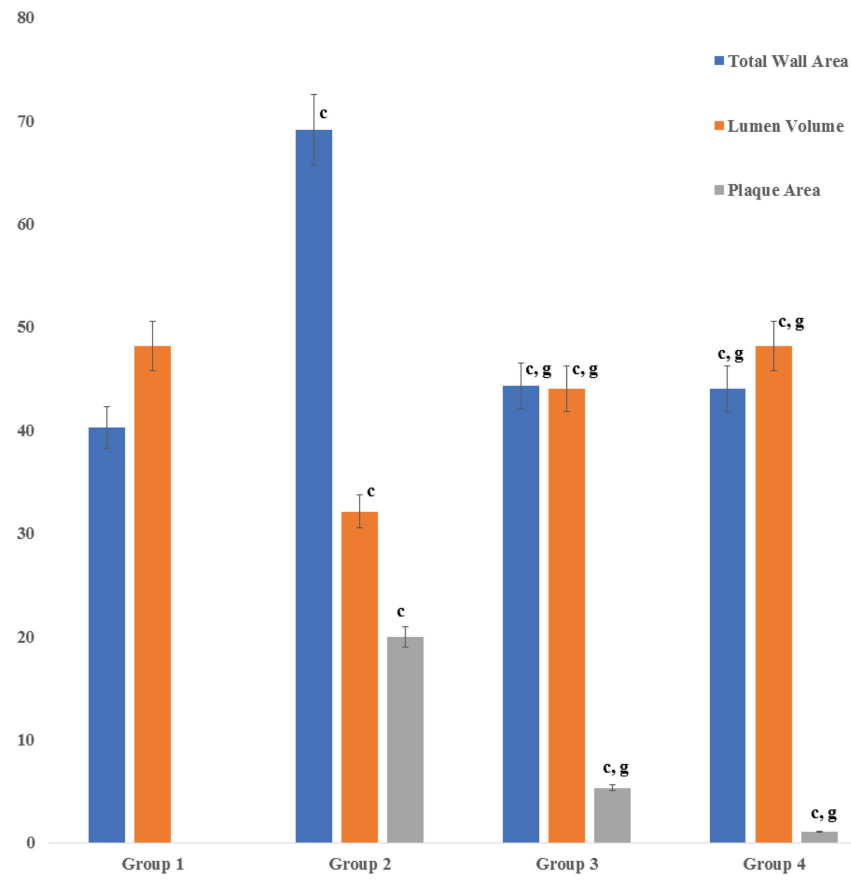

Figure 7: Planimetric study of aorta of the treatment and control groups.

of ADMET for curcumin along with toxicity as per the five rules of Lipinski. Further gastrointestinal absorption can be predicted by BIOLED-Egg assessment, which shows the suitability of the gastrointestinal absorption of curcumin and lipophilicity shown by the graphical criteria [12]. It is a well-established fact that bioavailability and bioreactivity of individual compounds depend on the crossing capability of the BBB, which make hurdles to accomplish the target. The gastrointestinal absorption depends on the lipophilicity and hydrophilicity of compounds as per the interactions of the existing functional groups, as well as availability of the lone pair of electrons [28]. Based on the integrative omics assessments, curcumin has potential interactive capabilities with HMG-CoA reductase and characteristics of API. Beside this, in-vivo assessments show that a high fat diet and cholesterol supplementation caused hypercholesterolemia and deposition of excess cholesterol content in liver as availability of reactant (high fat diet) which promoted cholesterol biosynthesis and least consumption (extra supplementation) [29,30]. However, the treatments of curcumin and atorvastatin caused significant reductions in TC, TG, LDL cholesterol, and VLDL cholesterol, along with the dyslipidemia indices of the AIP, Castelli index-I, and Castelli index-II. The kind of reductions in the lipid profile indicates that curcumin has the capacity to subside the cholesterol biosynthesis as revealed by several studies [31]. Accordingly, the data of molecular docking showed the interactive capability with $\mathrm{HMG}-\mathrm{CoA}$ reductase which is a key enzyme of cholesterol biosynthesis. The subsided HMG-CoA reductase directly hampered cholesterol biosynthesis, as reported by several studies, and the mechanism of action of the statins [32]. Supportively, the reductions in dyslipidemia indices such as AIP, CRI-I, and CRIII indicated the promoted reverse cholesterol transport described by the packing of different lipoproteins and reshuffling of excess peripheral cholesterol $[33,34]$. Subsequently, the treatments caused regressions in atherosclerotic plaque and total wall area along with increased lumen volume which depicted the elevated reverse cholesterol transport $[35,36]$. The regression of atherosclerotic plaque was promoted through several pathways, but the HMGCoA reductase inhibition is one of the established facts [37]. The results of in-vivo and integrative omics have proved that curcumin can serve as a potent antiatherosclerotic agent.

\section{CONCLUSION}

Based on the results of the integrative omics assessments and the in-vivo study of animal model, curcumin showed that it can serve as an API, which may be used as a therapeutic molecule for therapeutics of hypercholesterolemia.

\section{ACKNOWLEDGMENT}

The authors sincerely acknowledge the Department of Zoology for providing the essential facility and CSIR for providing fellowship.

\section{CONFLICT OF INTEREST}

The authors declared that there is no conflict of interest regarding this study.

\section{FUNDING}

The study was not supported by any kind of research grant.

\section{ETHICAL APPROVAL}

The protocols of the current study were approved by the Institutional Animal Ethical Committee, Department of Zoology, JNVU, Jodhpur (Reg. No.1646/GO/a/12/CPCSEA valid up to 27.03.23).

\section{AUTHOR CONTRIBUTIONS}

Priyanka Riyad: in-vivo experimentations; Ashok Purohit: conceptualization and supervision; Karishma Sen: integrative omics assessment; Heera Ram: conceptualization, editing, and correspondence. All authors read the final draft and approved it for communication.

\section{REFERENCES}

1. Nelson KM, Dahlin JL, Bisson J, Graham J, Pauli GF, Walters MA. The essential medicinal chemistry of curcumin. J Med Chem 2017;60(5):1620-37.

2. Hatcher H, Planalp R, Cho J, Torti FM, Torti SV. Curcumin: from ancient medicine to current clinical trials. Cell Mol Life Sci 2008;65(11):1631-52.

3. Ghosh S, Bhattacharyya S, Rashid K, Sil PC. Curcumin protects rat liver from streptozotocin-induced diabetic pathophysiology by counteracting reactive oxygen species and inhibiting the activation of p53 and MAPKs mediated stress response pathways. Toxicol Rep 2015;2:365-76.

4. Alappat L, Awad AB. Curcumin and obesity: evidence and mechanisms. Nutr Rev 2010;68(12):729-38.

5. Goel A, Kunnumakkara AB, Aggarwal BB. Curcumin as "curecumin": from kitchen to clinic. Biochem Pharmacol 2008;75(4):787-809.

6. Stanic Z. Curcumin, a compound from natural sources, a true scientific challenge - a review. Plant Foods Hum Nutr 2017;72(1):1-12.

7. Madhavi Sastry G, Adzhigirey M, Day T, Annabhimoju R, Sherman W. Protein and ligand preparation: parameters, protocols, and 
influence on virtual screening enrichments. J Comput Aided Mol Des 2013;27(3):221-34.

8. Chandran M, George S, Santhalingam K, Gangwar P, Krishnakumar K. Molecular docking studies of $2 \alpha$-hydroxyursolic acid derivatives for hypercholesterolemia. Int J PharmTech Res 2011;3(3):1576-81.

9. Jasmine JM, Vanaja R. In silico analysis of phytochemical compounds for optimizing the inhibitors of HMG CoA reductase. J Appl Pharm Sci 2013;3(9):43-7.

10. Guan L, Yang H, Cai Y, Sun L, Di P, Li W, et al. ADMET-score-a comprehensive scoring function for evaluation of chemical druglikeness. Medchemcomm 2019;10(1):148-57.

11. Tian S, Wang J, Li Y, Li D, Xu L, Hou T. The application of in silico drug-likeness predictions in pharmaceutical research. Adv Drug Deliv Rev [Internet] 2015;86:2-10.

12. Daina A, Zoete V. A Boiled-egg to predict gastrointestinal absorption and brain penetration of small molecules. ChemMedChem 2016;11:1117-21.

13. Ram H, Jatwa R, Purohit A. Antiatherosclerotic and cardioprotective potential of acacia senegal seeds in diet-induced atherosclerosis in rabbits. Biochem Res Int 2014;2014:1-9.

14. Hartvigsen K, Binder CJ, Hansen LF, Rafia A, Juliano J, Horkko S, et al. A diet-induced hypercholesterolemic murine model to study atherogenesis without obesity and metabolic syndrome. Arterioscler Thromb Vasc Biol 2007;27(4):878-85.

15. Rashid K, Sil PC. Curcumin enhances recovery of pancreatic islets from cellular stress induced inflammation and apoptosis in diabetic rats. Toxicol Appl Pharmacol 2015;282(3):297-310.

16. Khan TJ, Ahmed YM, Zamzami MA, Mohamed SA, Khan I, Baothman OAS, et al. Effect of atorvastatin on the gut microbiota of high fat dietinduced hypercholesterolemic rats. Sci Rep [Internet] 2018;8(1):1-9.

17. Underwood W. AVMA guidelines for the euthanasia of animals: 2020 edition. ESC Cardio Med. American Veterinary Medical Association, Schaumburg, IL, pp 33-4, 2020.

18. Ram H, Ram H, Jatwa R, Purohit A. Antiatherosclerotic and cardioprotective potential of Acacia senegal seeds in diet- induced atherosclerosis in rabbits antiatherosclerotic and cardioprotective potential of Acacia senegal seeds in diet-induced atherosclerosis in rabbits. Biochem Res Int 2014;2014:1-6

19. Abel LL, Levy BB, Brodie BB, Kendall FE, Abell L, Levy BB, et al. A simplified method for the estimation of total cholesterol in serum and demonstration of its specificity. J Biol Chem 1952;195(1):357-66.

20. Bucolo G, David H. Quantitative determination of serum triglycerides by the use of enzymes. Clin Chem 1973;19(5):476-82.

21. Moshides JS. Kinetic Enzymatic method for automated determination of HDL cholesterol in plasma. Clin Chem Lab Med 1987;25(9):583-8.

22. Vujovic A, Kotur-Stevuljevic J, Spasic S, Bujisic N, Martinovic J, Vujovic M, et al. Evaluation of different formulas for LDL-C calculation. Lipids Health Dis 2010;9:1-9.

23. Kamoru AA, Japhet OM, Adetunji AD, Musa MA, Hammed OO, Akinlawon AA, et al. Castelli risk index, atherogenic index of plasma, and atherogenic coefficient: emerging risk predictors of cardiovascular disease in HIV-treated patients. Int J Clin Trials Case Stud [Internet] 2017;2(3):8-15. Available via http://scholarsmepub.com/sjmps/ Website:http://scholarsmepub.com/
24. Mani JS, Johnson JB, Steel JC, Broszczak DA, Neilsen PM, et al. Natural product-derived phytochemicals as potential agents against coronaviruses: a review. Virus Res 2020;284:1-16.

25. Mohammed A, Islam MS. Spice-derived bioactive ingredients: potential agents or food adjuvant in the management of diabetes mellitus. Front Pharmacol 2018;9:1-28.

26. Hutchins KM. Functional materials based on molecules with hydrogen-bonding ability: applications to drug co-crystals and polymer complexes. R Soc Open Sci 2018;5(6):1-16.

27. Patel BD, Bhadada SV, Ghate MD. Design, synthesis and anti-diabetic activity of triazolotriazine derivatives as dipeptidyl peptidase-4 (DPP4) inhibitors. Bioorg Chem 2017;72:345-58.

28. Daina A, Michielin O, Zoete V. SwissADME: a free web tool to evaluate pharmacokinetics, drug-likeness and medicinal chemistry friendliness of small molecules. Sci Rep 2017;7:1-13.

29. Scallen TJ, Sanghvi A. Regulation of three key enzymes in cholesterol metabolism by phosphorylation/dephosphorylation. Proc Natl Acad Sci U S A 1983;80(9):2477-80.

30. Okoduwa SIR, Umar IA, James DB, Inuwa HM. Appropriate insulin level in selecting fortified diet-fed, streptozotocin-treated rat model of type 2 diabetes for anti-diabetic studies. PLoS One 2017;12(1):1-21.

31. Suganya S, Nandagopal B, Anbarasu A. Natural inhibitors of HMGCoA reductase - an insilico approach through molecular docking and simulation studies. J Cell Biochem 2017;118(1):52-7.

32. Holdgate GA, Ward WHJ, Mctaggart F, Park A, Sk C. Molecular mechanism for inhibition of reductase by rosuvastatin. Biochem Soc Trans 2003;31:528-31.

33. Turner S, Voogt J, Davidson M, Glass A, Killion S, Decaris J, et al Measurement of reverse cholesterol transport pathways in humans: in vivo rates of free cholesterol efflux, esterification, and excretion. J Am Heart Assoc 2012;1(4):1-11.

34. Olamoyegun M, Oluyombo R, Asaolu S. Evaluation of dyslipidemia, lipid ratios, and atherogenic index as cardiovascular risk factors among semi-urban dwellers in Nigeria. Ann Afr Med 2016;15(4):194-9.

35. Páramo JA, Civeira F. Is regression of atherosclerotic plaque possible? Clin Investig Arterioscler [English Ed (Internet)] 2017;29(1):46-50.

36. Kalanuria AA, Nyquist P, Ling G. The prevention and regression of atherosclerotic plaques: emerging treatments. Vasc Health Risk Manag 2012;8(1):549-61.

37. Elshazly MB, Stegman B, Puri R. Regression of coronary atheroma with statin therapy. Curr Opin Endocrinol Diabetes Obes 2016;23(2):131-7.

How to cite this article:

Riyad P, Purohit A, Sen K, Ram H. Atherosclerotic plaque regression and $\mathrm{HMG}-\mathrm{CoA}$ reductase inhibition potential of curcumin: An integrative omics and in-vivo study. J Appl Biol Biotech 2022; 10(01):129-135. 